\title{
SOUND TRANSMISSION FROM A TUBE WITH FLOW*
}

\author{
BY G. F. CARRIER (Harvard University)
}

1. Introduction. The increasing technological importance of thermo-acoustic phenomena in combustion chambers and other apparatus leads naturally to the need for understanding the transmission and reflection of acoustic waves at the inlet and exit sections of tubes through which a gas is flowing at moderate Mach number. In this paper, we treat this question for the inviscid perfect gas. The flow fields adopted are the simplest which are reasonable approximations to interesting physical situations and which lead to tractable problems. The problems are resolved by an extension of the work of Schwinger and Levine [1] (their work is essentially the $M=0$ problem), and, in some interesting cases, our results can be expressed directly in terms of theirs. The modifications in these cases are reminiscent of subsonic aerodynamic results obtained using the Prandtl-Glauert method.

2. Formulation of the problems. The configuration to be treated here is depicted in Fig. (2.1). The steady flow through and about the tube is characterized by the specifi-

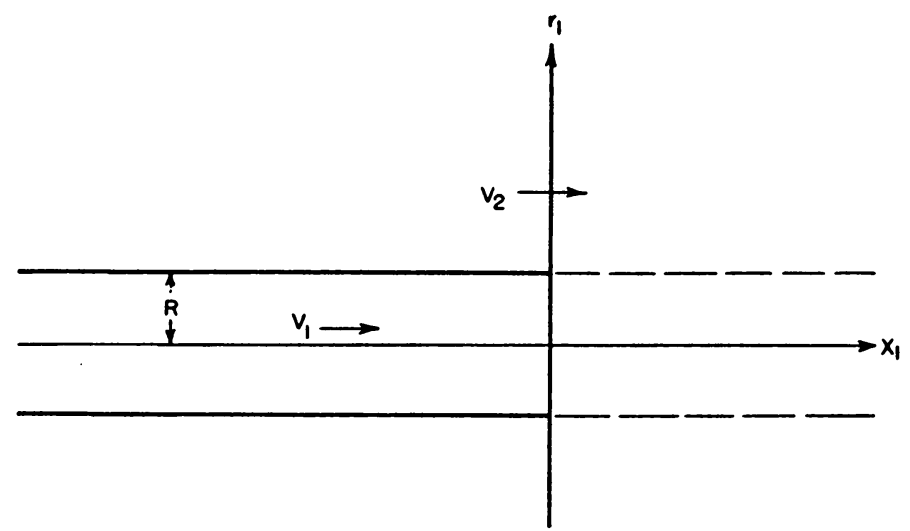

Fig. 2.1. Geometry of sound transmission problem.

cation of the thermodynamic state variables and the velocity field; the velocity field, in turn, is characterized by a single non-vanishing velocity component in the $x_{1}$ direction. This velocity has the value $v_{1}$ for $r_{1}<R$ and $v_{2}$ for $r_{1}>R$. In two of the problems of interest $v_{1}=v_{2}$ and the state variables are the same in each region. In the third $v_{1}>v_{2}$ and the state variables may differ in the two domains. The first two cases are distinguished according to whether $v_{1}$ is greater or less than zero.

If we restrict our analysis to small disturbances, assuming a perfect, inviscid, nonheat conducting gas, the equations governing the propagation of the disturbance in each region take the well known form

$$
\begin{gathered}
\rho \operatorname{div} \nabla^{\prime}+\rho_{t}^{\prime}+v \rho_{x_{1}}^{\prime}=0, \\
\rho\left(\nabla_{t}^{\prime}+v \nabla_{x}^{\prime}\right)+\operatorname{grad} p^{\prime}=0 .
\end{gathered}
$$

*Received March 11, 1955. 
Here, the primed quantities $\left(\nabla^{\prime}, \rho^{\prime}, p^{\prime}\right)$ are the perturbations in velocity, density and pressure, $\rho$ is the steady state (constant) density, and the isentropic pressure-density law implies that $p^{\prime}=a^{2} \rho^{\prime}$ where $a$ is the acoustic speed associated with the steady flow state; $x_{1}, r_{1}$ are the space coordinates. Each of these quantities will require the subscript 1 or 2 to distinguish the domain to which it applies when we discuss the flow in which $v_{1} \neq v_{2}$.

It is convenient to introduce the following notation: $\mathbf{v}^{\prime}=a R^{\prime} \operatorname{grad} \phi, R^{\prime}=$ $R\left(1-M^{2}\right)^{1 / 2}, s=x_{1} / R^{\prime}, y=r_{1} / R, k^{2}=\omega^{2} R^{2} / a^{2}\left(1-M^{2}\right)$, and $\phi=\psi(s, y) \exp \{-i \omega[t+$ $\left.\left.M x_{1} / a\left(1-M^{2}\right)\right]\right\}$. It follows from these and the foregoing that

and

$$
\Delta \psi+k^{2} \psi=0
$$

$$
p^{\prime}=-\rho a^{2}\left(M \psi_{s}-i k \psi\right) \exp \left\{-i \omega\left[t+M x_{1} / a\left(1-M^{2}\right)\right]\right\},
$$

where $\Delta$ is the cylindrical coordinate Laplace operator. ${ }^{1}$ The boundary conditions we shall use differ in the three problems. If problem $\mathrm{I}$ is characterized by $v_{1}=v_{2}>0$, the essential information is: $p^{\prime}(x, R, t)$ is continuous on $s>0, \psi_{\nu}(s, 1)=0$ on $s<0$. Note that, as in oscillating airfoil theory, one can anticipate that $\psi(s, 1)$ will not be continuous for $s>0$. In problem II, $v_{1}=v_{2}<0$ and both $p^{\prime}$ and $\psi$ are continuous on $y=1, s>0$; again, $\psi_{y}(s, 1)=0$ when $s<0$. For problem III, with $v_{1}$ and $v_{2}$ positive but not equal, the boundary conditions are the same as those of problem I.

3. The continuous flow problems. Problems I and II differ only in minor respects from the problem treated in [1]. This enables us to reduce these problems to such a form that the results of interest can be obtained directly in terms of quantities computed in that paper. The ordinary differential equation which $\chi$, the Fourier transform of $\psi$, [i.e. $\chi(\xi, y)=\int_{-\infty}^{\infty} \psi(x, y) \exp (-i \xi x) d x$ ], must obey is

$$
y^{-1}\left(y \chi_{\nu}\right)_{\psi}+\left(k^{2}-\xi^{2}\right) \chi=0 .
$$

The appropriate solution (obeying the radiation condition) is

and

$$
\chi=A(\xi) J_{0}\left(\left[k^{2}-\xi^{2}\right]^{1 / 2} y\right), \quad y<1
$$

$$
\chi=B(\xi) H_{0}^{(1)}\left(\left[k^{2}-\xi^{2}\right]^{1 / 2} y\right), \quad y>1 .
$$

The boundary conditions can be written concisely if we define $h(\xi)=(i k-i M \xi)$ $[\chi(\xi, 1-)-\chi(\xi, 1+)]$. The pressure boundary condition then merely implies that $h(\xi)$ is the transform of a function, $H(x)$, which is zero for $x>0$. We also note that $w(\xi)=\chi_{v}(\xi, 1)$ is the transform of a function, $W(x)$ which vanishes for $x<0$. Now

and

$$
h(\xi)=i(k-M \xi)\left\{A(\xi) J_{0}\left(\left[k^{2}-\xi^{2}\right]^{1 / 2}\right)-B(\xi) H_{0}^{(1)}\left(\left[k^{2}-\xi^{2}\right]^{1 / 2}\right)\right\}
$$

$$
w(\xi)=-A\left(k^{2}-\xi^{2}\right)^{1 / 2} J_{1}\left(\left[k^{2}-\xi^{2}\right]^{1 / 2}\right)=-B\left(k^{2}-\xi^{2}\right)^{1 / 2} H_{1}^{(1)}\left(\left[k^{2}-\xi^{2}\right]^{1 / 2}\right) .
$$

Eliminating $A$ and $B$, we obtain (using $\left.J_{1}(\zeta) H_{0}^{(1)}(\zeta)-J_{0}(\zeta) H_{1}^{(1)}(\zeta)=2 i / \pi \zeta\right)$,

$$
-\pi i\left(k^{2}-\xi^{2}\right) H_{1}^{(1)}\left(\left[k^{2}-\xi^{2}\right]^{1 / 2}\right) J_{1}\left(\left[k^{2}-\xi^{2}\right]^{1 / 2}\right) h(\xi)=2 i(k-M \xi) w(\xi) .
$$

Our foregoing remarks imply that $h(\xi)$ is analytic when $\operatorname{Im} \xi>0$ and that $w(\xi)$ is analytic

${ }^{1}$ We consider only problems in which the incident wave is a plane wave in the tube normal to its axis and approaching the origin. 
when $\operatorname{Im} \xi<0$. The domains of analyticity of these functions can be extended if we associate with $k$ a complex value ( $\operatorname{Im} k=\epsilon>0)$. When this is done, we can anticipate that $h, w$, and the coefficient of $h$ in (3.3) will be analytic in the strip $|\operatorname{Im} \xi|<\epsilon$. This is demonstrated in detail in [1]. We now write ${ }^{2}$

$$
L(\xi)=\pi i H_{1}^{(1)}\left(\left[k^{2}-\xi^{2}\right]^{1 / 2}\right) J_{1}\left(\left[k^{2}-\xi^{2}\right]^{1 / 2}\right)=L_{+}(\xi) / L_{-}(\xi),
$$

where $L_{+}$and $L_{-}$are respectively analytic above $-i \epsilon$ and below $i \epsilon$. One can now apply the conventional arguments of the Wiener-Hopf technique to find $h(\xi)$, and these arguments lead to the conclusion that each side of the equation

$$
-\left(k^{2}-\xi^{2}\right) L_{+}(\xi) h(\xi)=2 i(k-M \xi) L_{-}(\xi) w(\xi)
$$

is the analytic continuation of the other, and that each represents an entire function of $\xi$.

When $M>0$ (the exit problem), it is required that $H(x) \rightarrow 0$ as $x \rightarrow 0-$ and we can anticipate that $H(x) \sim|x|^{1 / 2}$ in this neighborhood. It was shown in [1] that $L_{+}(\xi) \sim \xi^{-1 / 2}$ for large $\xi$ (with $\operatorname{Im} \xi>0$ ). It follows that the entire function, $\left(\xi^{2}-\right.$ $\left.k^{2}\right) L_{+}(\xi) h(\xi)$, is a constant (because it is bounded at $\infty$ ).

When $M<0$, the situation is different. In this case, instead of demanding that $h(x) \rightarrow 0$ as $x \rightarrow 0-$, we require that $\psi$ be continuous on the extension of the tube. This implies that $-i h /(k-M \xi)$ be the transform of a function which behaves like $x^{1 / 2}$ near $x=0$ and, in this case, the entire function $\left(k^{2}-\xi^{2}\right) L_{+}(\xi) h(\xi)$, is a constant multiple of $(k-M \xi)$. One also can see this by noting that, in the entrance case, the radial velocity perturbation near the edge must behave like $x^{-1 / 2}$; then, since $L_{-}(\xi) \sim \xi^{1 / 2}$ (see [1]), the foregoing result ensues.

We are primarily interested in the reflection coefficient associated with the tube end. Since the pressure field in the tube for large negative $x$ must be of the form $H(x) \sim$ $\exp (i k s)+N \exp (-i k s)$, it follows that the reflection coefficient $N$ is the ratio of the residues of $h(\xi) \exp (i \xi s)$ at $-k$ and $+k$, respectively (i.e. $N=-L_{+}(k) / L_{+}(-k)$ for the exit problem and $N=-[(1+M) /(1-M)] L_{+}(k) / L_{+}(-k)$ for the inlet problem, where $M<0$ ). For the exit problem, these residues are precisely those computed in [1]. Thus our $N\left[(\omega R / a)(1-M)^{1 / 2}\right]$ is precisely the $R$ of [1]. Note that this is the reflection coefficient for the pressure field (and, as it happens, for the velocity field, too) but not for the potential.

For the inlet flow, on the other hand, the residues of $h(\xi) \exp (i \xi s)$ at $\pm k$ are not the same as those of the exit flow but rather those of this expression multiplied by $i(k-M \xi)$. Thus, for $M<0$, the reflection coefficient $N^{\prime}(k)$ is given by $[(1+M) /(1-$ $M)] N(k)$.

It is interesting to note that the reflection at the inlet end of the tube is much poorer than that at the exit end. This is most readily rationalized by noting that, at the inlet end, the incident wave "sees" a much smaller wave length to radius ratio. This is not an adequate basis for an estimate, however, because the non-convective theory gives a quadratic dependence on this ratio for small frequency whereas here the dependence is stronger and, in particular, $N$ is not unity for $\omega=0$.

The major result is to notice that for low frequencies, a moving tube has much greater radiation losses from an inlet end than it does in a stationary situation but that the losses at the exit are only affected when $M$ gets reasonably close to unity.

TThe notation is nearly identical with that of [1] in order that the reader may readily translate the thorough discussion of that paper into the straightforward generalization of this section. 
Figures (3.1) and (3.2) are graphs of $N$ and $l / R$ where $N=-|N| \exp (2 i k l / R)$. These are taken from [1].

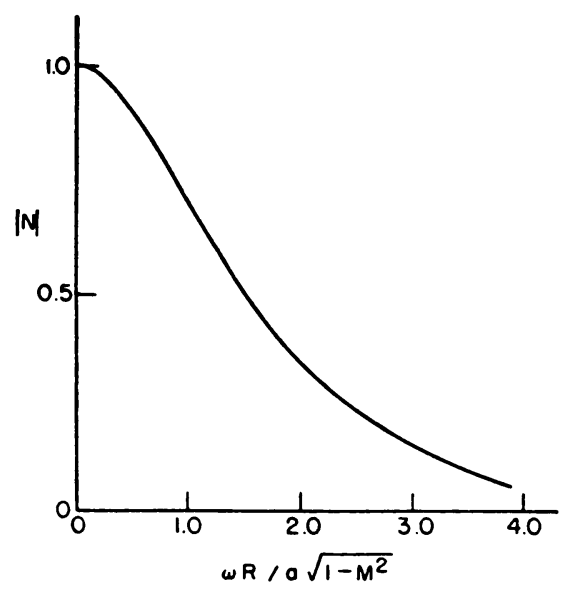

Fig. 3.1.

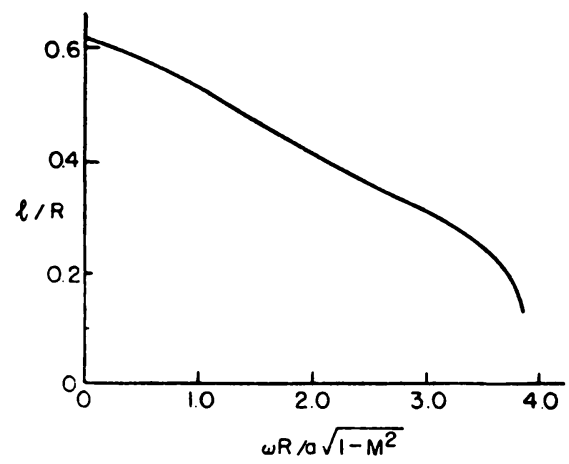

Frg. 3.2.

4. The jet flow. In this section we consider the algebraically more difficult problem where $M_{1} \neq M_{2}$ and $a_{1} \neq a_{2}$. In this problem, it is more convenient to distort the coordinate system uniformly (i.e. we use the same scale for $x$ and $r$ ) and we introduce the notation: $s=x / R, y=r / R, m_{i}=v_{i} / a_{i}, k_{i}=\omega R / a_{i}, \beta_{i}^{2}=1-M_{i}^{2}$ and $\nabla_{i}=a_{i} \operatorname{grad}$ $\phi_{i}(s, y) \exp (-i \omega t)$. The differential equations governing the $\phi_{i}$ become (proceeding from Eqs. (2.1) and (2.2))

$$
\begin{aligned}
& \Delta \phi_{1}-\left(M_{1} \frac{\partial}{\partial s}-i k_{1}\right)^{2} \phi_{1}=0, \\
& \Delta \phi_{2}-\left(M_{2} \frac{\partial}{\partial s}-i k_{2}\right)^{2} \phi_{2}=0 .
\end{aligned}
$$

If we again introduce the Fourier transforms with regard to $x$ of the $\phi_{i}$, call them $\chi_{i}(\xi, y)$, and integrate the ordinary differential equations which govern the $\chi_{i}(\xi, y)$, we obtain

$$
\begin{aligned}
& \chi_{1}(\xi, y)=A(\xi) J_{0}\left(z_{1} y\right), \\
& \chi_{2}(\xi, y)=B(\xi) H_{0}^{(1)}\left(z_{2} y\right),
\end{aligned}
$$

where $z_{i}^{2}=\left(k_{i}-M_{i} \xi\right)^{2}-\xi^{2}$. The velocity at $y=1, W(s)$ has the transform

$$
w(\xi)=-z_{1} A J_{1}\left(z_{1}\right)=-z_{2} B H_{1}^{(1)}\left(z_{2}\right)
$$

and the jump ${ }^{3}$ in pressure at $y=1, H(s)$, has the transform

$$
h(\xi)=\left(k_{1}-M_{1} \xi\right) A J_{0}\left(z_{1}\right)-\left(k_{2}-M_{2} \xi\right) B H_{0}^{(1)}\left(z_{2}\right) .
$$

Equations (4.5) and (4.6) may be combined to eliminate $A$ and $B$ and to obtain

$$
z_{1}^{2} L(\xi) h(\xi)=w(\xi)\left[\alpha-\left(M_{1}+M_{2} \beta_{1} / \beta_{2}\right) \xi\right],
$$

This is the dimensionless jump $\left(p_{1}^{\prime}-p_{2}^{\prime}\right) / p_{1}$. Note that $p_{1}=p_{2}$ for equilibrium of the steady flow. 
where

$$
L(\xi)=\frac{\left[\alpha-\left(M_{1}+M_{2} \beta_{1} / \beta_{2}\right) \xi\right]\left(z_{2} / z_{1}\right) J_{1}\left(z_{1}\right) H_{1}^{(1)}\left(z_{2}\right)}{\left(k_{2}-M_{2} \xi\right) z_{1} H_{0}^{(1)}\left(z_{2}\right) J_{1}\left(z_{1}\right)-\left(k_{1}-M_{1} \xi\right) z_{2} H_{1}^{(1)}\left(z_{2}\right) J_{0}\left(z_{1}\right)}
$$

and where $\alpha$ is so chosen that $L(\xi)$ is finite at that zero of the denominator of Eq. (4.8) which, when states 1 and 2 are identical, is located at $\xi=k_{2} / M_{2}$.

It is convenient to factor $L(\xi)$ in the following manner.

$$
\begin{aligned}
L_{1}(\xi) & =\pi i J_{1}\left(z_{1}\right) H_{1}^{(1)}\left(z_{1}\right), \quad L^{*}(\xi)=L / L_{1}, \\
= & \frac{-i\left[\alpha-\left(M_{1}+M_{2} \beta_{1} / \beta_{2}\right)\right] z_{2} H_{1}^{(1)}\left(z_{2}\right)}{\pi\left[\left(k_{1}-M_{1} \xi\right) z_{1} J_{0}\left(z_{1}\right) H_{1}^{(1)}\left(z_{1}\right) z_{2} H_{1}^{(1)}\left(z_{2}\right)-\left(k_{2}-M_{2} \xi\right) H_{0}^{(1)}\left(z_{2}\right) z_{1}^{2} H_{1}^{(1)}\left(z_{1}\right) J_{1}\left(z_{1}\right)\right]}
\end{aligned}
$$

and the factor $L_{1}(\xi)$ is precisely the Schwinger-Levine $L(\zeta)$ where $\zeta=\beta_{1} \xi+k_{1}$.

The solution of Eq. (4.7) for $h(\xi)$ now requires that we split $L(\xi)$ [and hence both $L_{1}(\xi)$ and $\left.L^{*}(\xi)\right]$ into factors $L_{+}(\xi), L_{-}(\xi)$ which are analytic above $-i \epsilon_{2}$ and below $i \epsilon_{1}$, respectively (here $\epsilon_{i}$ is the imaginary part of the root of $z_{i}=0$ when $\operatorname{Im} k_{2}=\epsilon$ ).

The reflection coefficient will be given again by the ratio of the amplitudes of the returning and the outgoing waves in the tube. The inversion integral for the pressure $H(x)$ in the tube (as $x \rightarrow-\infty$, the external pressure tends to 0 so the interior pressure becomes the pressure jump $H(x)$ ) is given by

$$
H(x)=\frac{1}{2 \pi} \int_{-\infty}^{\infty}\left[L_{+}(\xi)\right]^{-1} \exp (i \xi x)\left[z_{1}(\xi)\right]^{-2} d \xi
$$

and the asymptotic result for $-x \gg 1$ is given by the residues at the zeros of $z_{1}^{2}$. That is, the reflection coefficient is again $L_{+}\left(-b_{2}\right) / L_{+}\left(b_{1}\right)$ where $-b_{2}$ and $b_{1}$ are the zeros of $z_{1}^{2}$. However, $L_{+}$is composed of two factors, the $L_{+}(\xi)$ associated with $L_{1}$ as used in the .foregoing and the factor $L_{+}^{*}$. The Cauchy integral formula gives $L_{+}^{*}$ in the form

$$
\ln L_{+}^{*}(t)=\frac{1}{2 \pi i} \int_{-\infty}^{\infty}(\xi-t)^{-1} \ln L^{*}(\xi) d \xi
$$

and, in particular, we define the quantity $Q$ so that

$$
\ln Q=\ln \left(L_{+}^{*}\left(-b_{2}\right) / L_{+}^{*}\left(b_{1}\right)\right)=\frac{-k_{1}}{\pi i \beta_{1}^{2}} \int_{-\infty}^{\infty} \frac{\ln L^{*}(\xi)}{\left(\xi-b_{1}\right)\left(\xi+b_{2}\right)} d \xi
$$

where the path of integration is indented to pass below $-b_{2}$ and $b_{1}$. When $k_{1}=k_{2}$, $M_{1}=M_{2}, L^{*}(\xi)$ is identically unity and the contribution of (4.11) is $Q=1$. For other cases, however, $L^{*}(\xi) \rightarrow 1$ as $\xi \rightarrow \pm \infty$ and the convergence of the integral is assured. The reflection coefficient in such cases is given by the product

$$
Q\left(k_{1}, k_{2}, M_{1}, M_{2}\right) N\left(k_{1} / \beta_{1}\right) \text {. }
$$

Again one can use the results of [1] to find $N$ but the determination of $Q$ requires the numerical evaluation of the integral of (4.11) for those values of the four independent parameters $a_{1}, a_{2}, M_{1}, M_{2}$ which are of interest.

\section{BIBLIOGRAPHY}

1. H. Levine and J. Schwinger, On the radiation of sound from an unflanged circular pipe, Phys. Rev. 73 (1948) 